\title{
Vorinostat enhances protein stability of p27 and p21 through negative regulation of Skp2 and Cks1 in human breast cancer cells
}

\author{
NORIHISA UEHARA, KATSUHIKO YOSHIZAWA and AIRO TSUBURA \\ Second Department of Pathology, Kansai Medical University, Moriguchi-shi, Osaka 570-8506, Japan
}

Received February 10, 2012; Accepted March 14, 2012

DOI: $10.3892 /$ or.2012.1758

\begin{abstract}
Vorinostat is a histone deacetylase inhibitor that blocks cancer cell proliferation through the regulation of cyclin-dependent kinase inhibitors. We, herein, examined the involvement of S-phase kinase-associated protein 2 (Skp2) and cyclin-dependent kinase subunit 1 (Cks1), the components of the $\mathrm{SCF}^{\text {Skp2-Cks1 }}$ (Skp1/Cul1/F-box protein) ubiquitin ligase complex, in the regulation of p27 and p21 during vorinostatinduced growth arrest of MDA-MB-231 and MCF-7 human breast cancer cells. Vorinostat significantly reduced BrdU incorporation in MDA-MB-231 and MCF-7 cells, which was associated with increased p27 and p21 protein levels without concomitant induction of p27 mRNA. Vorinostatinduced accumulation of $\mathrm{p} 27$ and $\mathrm{p} 21$ proteins was inversely correlated with the mRNA and protein levels of Skp2 and Cks1. Cycloheximide chase analysis revealed that vorinostat increased the half-life of p27 and p21 proteins. The accumulation of p27 and p21 proteins was attenuated by forced expression of Skp2 and Cks1, which conferred resistance to the vorinostat-induced $\mathrm{S}$-phase reduction. These results suggest that vorinostat-induced growth arrest may be in part due to the enhanced protein stability of p27 and p21 through the downregulation of Skp2 and Cks1.
\end{abstract}

\section{Introduction}

Cell cycle progression is tightly controlled by the activities of cyclin-dependent kinases (CDKs) and CDK inhibitors (CKIs), such as p27 and p21 (1). Timely degradation of these proteins is required for proper cell-cycle progression. S-phase kinase associated protein $2(\mathrm{Skp} 2)$ and cyclin-dependent kinase subunit $1(\mathrm{Cks} 1)$ are the subunits of the $\mathrm{SCF}^{\text {Skp2-Cks1 }}$ (Skp1/Cul1/F-box protein) ubiquitin ligase complex that are responsible for S-phase entry through the ubiquitination of p27 and p21, which is followed by their degradation by the

Correspondence to: Dr Norihisa Uehara, Second Department of Pathology, Kansai Medical University, 10-15 Fumizono-cho, Moriguchi-shi, Osaka 570-8506, Japan

E-mail: ueharan@takii.kmu.ac.jp

Key words: vorinostat, $\mathrm{SCF}^{\text {Skp2-Cks1 }}$ ubiquitin ligase, p27, p21, breast cancer proteasome pathway (2). Many cancer cells exhibit abnormal proliferation that is attributed to aberrant cell cycle progression. Numerous studies have reported the loss of p27 in a wide variety of malignancies including breast cancer $(3,4)$. The loss of p27 was due to accelerated degradation by the ubiquitin-proteasome pathway, rather than transcriptional repression. In fact, overexpression of Skp2 and/or Cks1 has been found in breast cancer tissues and is an independent prognostic marker for disease-free and overall survival and is associated with aggressiveness (5-9). In cell culture, higher expression levels of Skp2 were found in estrogen receptor (ER)/Her2-negative aggressive breast cancer cells, such as MDA-MB-231, as compared to MCF-7 ER-positive breast cancer cells $(6,7)$.

Vorinostat is a potent histone deacetylase inhibitor (HDACi) that exerts antitumor effects by inducing differentiation, growth arrest and/or apoptosis through acetylation modification of histones and/or non-histone proteins (10). Vorinostat induces cell-cycle arrest at the G1 or the G2/M boundary in a cell-type dependent and/or dose-related fashion (11). The transactivation of $C D K N 1 A$, which encodes $\mathrm{p} 21$, is commonly observed after HDACi treatment (12). In addition, induction of p27 in breast cancer cells by vorinostat has also been reported $(13,14)$. To date, several reports indicate that the downregulation of Skp2 by HDACi contributes to the stability of p27 protein. However, the effect of vorinostat on the expression of Skp2 and Cks1 in relation to the protein stability of p27 and p21 in breast cancer cells has not been fully elucidated. Therefore, MCF-7 and MDA-MB-231 cells were used in this study because of their different characteristics in terms of ER, Skp2 and Cks1 expression.

Here, we demonstrate that vorinostat decreases the levels of both Skp2 and Cks1 in human breast cancer cells, and that these decreases correlate with the accumulation of p27 and p21 protein. Our data imply that not only the transcriptional activation of $\mathrm{p} 21$, but also the enhanced protein stability of p27 and p21 through the modulation of Skp2 and Cks1, may contribute to the vorinostat-induced growth arrest of human breast cancer cells.

\section{Materials and methods}

Cell lines and materials. The human breast cancer cell lines MDA-MB-231 and MCF-7 were purchased from the American Type Culture Collection (Rockville, MD). Cells 
were maintained as previously described (15). Vorinostat (suberoylanilide-hydroxamic acid) was obtained from Selleck (Houston, TX). 5-bromo-2'-deoxyuridine (BrdU) and cycloheximide (CHX) were obtained from Sigma (St. Louis, MO) and Calbiochem (San Diego, CA), respectively.

BrdU incorporation analysis. The proliferation status of cells was monitored by assaying BrdU incorporation. Briefly, cells were seeded at $1 \times 10^{4}$ cells/well on 8 -well slide chambers in growth medium supplemented with $10 \%$ serum and incubated overnight. After exposure to vorinostat for the indicated amount of time, cells were labeled with $10 \mu \mathrm{M}$ BrdU for $1 \mathrm{~h}$. The cells were fixed with $10 \%$ buffered formalin for $10 \mathrm{~min}$ at room temperature, treated with $3 \%$ hydrogen peroxide for $10 \mathrm{~min}$, incubated with $4 \mathrm{~N} \mathrm{HCl}$ at $37^{\circ} \mathrm{C}$ for 20 min and digested with $0.5 \mathrm{mg} / \mathrm{ml}$ actinase at $37^{\circ} \mathrm{C}$ for $1 \mathrm{~min}$. Cells were blocked with $1 \%$ bovine serum albumin in TBST for $5 \mathrm{~min}$ and incubated with anti-BrdU antibody (BD Biosciences, San Jose, CA) at room temperature for $1 \mathrm{~h}$. The antigen-antibody complexes were detected by using an LSAB staining kit (Dako, Carpinteria, $\mathrm{CA}$ ) according to the manufacturer's instructions, and reaction products were visualized with 3-3'-diaminobenzidine tetrahydrochloride. BrdU-labeling indices were determined by counting >100 cells in 5 independent fields and scoring BrdUpositive cells as a percentage of the total cell number.

Quantitative real-time PCR analysis. mRNA expression was analyzed by performing quantitative real-time PCR with the iQ5 Optical System Software version 2.1 (Bio-Rad Laboratories, Hercules, CA). Total RNA was prepared by using an RNeasy Mini kit (Qiagen, Valencia, CA). The cDNA was then reversetranscribed from $1.0 \mu \mathrm{g}$ of RNA by using a SuperScript III first-strand cDNA synthesis kit (Invitrogen, Carlsbad, CA). The primers used were 5'-TGTCAAACGTGCGAGTGTCT-3' and 5'-CTCGCCTCTTCCATGTCTCT-3' for p27, 5'-GGAAG ACCATGTGGACCTGT-3' and 5'-GGATTAGGGCTTCC TCTTGG-3' for $p 21$, 5'-GCCCACGATCATTTATGGAC-3' and 5'-ATTGACAATGGGATCCGAAA-3' for $S k p 2$, 5'-TAG CCAAGCTGGTCCCTAAA-3' and 5'-GCCAGCTTCATTT CTTTGGT-3' for $C k s 1$, and 5'-AGAAAATCTGGCACCAC ACC-3' and 5'-AGAGGCGTACAGGGATAGCA-3' for $\beta$-actin. PCR amplification was performed in triplicate with SsoFast $^{\mathrm{TM}}$ EvaGreen ${ }^{\circledR}$ Supermix (Bio-Rad Laboratories). The PCR conditions were as follows: enzyme activation at $95^{\circ} \mathrm{C}$ for $30 \mathrm{sec}, 40 \mathrm{cycles}$ of $5 \mathrm{sec}$ at $95^{\circ} \mathrm{C}, 10 \mathrm{sec}$ at $60^{\circ} \mathrm{C}$. The expression was calculated by using the comparative threshold cycle (CT) method (16).

Western blot analysis. Western blots were performed as previously described (17). The following primary antibodies were used: anti-p27 and anti-p21 antibodies (Cell Signaling Technology, Beverly, MA); anti-Skp2 p45 (H-435) and HRP-conjugated anti-actin antibodies (C-11) (Santa Cruz Biotechnology, Santa Cruz, CA); anti-p19 (Skp1) antibody (BD Biosciences); and anti-Cks1 antibody (Zymed, Carlsbad, CA). The films were scanned and subjected to densitometric analyses with the ImageJ software developed at NIH.

Determination of protein half-life. For determination of the half-life of p27 and p21 protein, $1 \times 10^{5}$ cells were seeded on 60-mm culture dishes in growth medium supplemented with $10 \%$ serum and incubated overnight. Cells were cultured for $24 \mathrm{~h}$ in the presence or absence of vorinostat and then exposed to $10 \mu \mathrm{g} / \mathrm{ml} \mathrm{CHX}$. At the indicated times following $\mathrm{CHX}$ treatment, cells were lysed and protein levels were determined by western blot analysis. The films were scanned and subjected to densitometric analyses with ImageJ software.

Transient transfection. The skp2 and $c k s 1$ expression vectors (pcDNA-FLAG-Skp2 and pcDNA-FLAG-Cks1) were kindly provided by Dr Michele Pagano (New York University, NY, USA). Transient transfections were performed with Lipofectamine LTX reagent (Invitrogen) according to the manufacturer's recommendations. Cells were plated at $2 \times 10^{5}$ cells per well in 6-well culture plates and incubated overnight. The transfection was performed with $2 \mu \mathrm{g}$ of plasmid DNA mixed with Lipofectamine LTX reagent. Cells were incubated for $4 \mathrm{~h}$ with this mixture in $2 \mathrm{ml}$ antibiotic-free culture medium with $10 \%$ serum. Then, the medium was replaced with fresh culture medium containing $10 \%$ serum.

Cell-cycle analysis. Cell-cycle analysis was performed as previously described (18). Cells were plated at $5 \times 10^{5}$ cells $/ 100-\mathrm{mm}$ dish and incubated overnight. At $24 \mathrm{~h}$ after vorinostat treatment, cells were collected by trypsinization and fixed in $70 \%$ ethanol. The cells were collected by centrifugation at $600 \mathrm{x}$ g for $5 \mathrm{~min}$ at $4^{\circ} \mathrm{C}$ and washed twice with PBS. The cells were then resuspended in PBS containing $250 \mathrm{U} / \mathrm{ml}$ RNase for $20 \mathrm{~min}$ at room temperature and stained with $100 \mu \mathrm{g} / \mathrm{ml}$ propidium iodide (PI). The DNA content was examined with a FACSCalibur apparatus (Becton-Dickinson, San Jose, CA). Cell-cycle distribution was analyzed with CellQuest (BectonDickinson) and ModFit LT software (Verity, Topsham, ME).

Statistical analysis. All data are expressed as mean $\pm \mathrm{SD}$, and were analyzed with the Student's t-test. P-values $<0.05$ indicate statistically significant differences.

\section{Results}

Vorinostat blocks DNA synthesis in human breast cancer cells. The effect of vorinostat on DNA synthesis was monitored by a BrdU incorporation assay (Fig. 1). MDA-MB-231 and MCF-7 cells were treated with the half-maximal inhibitory concentration $\left(\mathrm{IC}_{50}\right.$ ) of vorinostat (MDA-MB-231; $5.0 \mu \mathrm{M}$ and MCF-7; $2.5 \mu \mathrm{M})$ (15) for varying periods of time $(\sim 72 \mathrm{~h})$. As shown in Fig. 1, the effect of vorinostat on the blockade of BrdU incorporation peaked at $24 \mathrm{~h}$ ( $\sim 95 \%$ in both cell lines), and it persisted until $72 \mathrm{~h}(\sim 88 \%$ in MDA-MB-231 and $\sim 84 \%$ in MCF-7), which was correlated with the acetylation status of histone $\mathrm{H} 3$ and growth inhibition (15).

Vorinostat induces post-translational accumulation of p27 and $p 21$. Although p27 and p21 protein are induced by vorinostat in several human breast cancer cell lines $(13,14)$, the relationship between mRNA expression and protein levels of p27 and p21 after vorinostat treatment has not been fully elucidated. Therefore, we confirmed the mRNA and protein level of p27 and p21 after vorinostat treatment in MDA-MB-231 and MCF-7 cells (Fig. 2). MDA-MB-231 cells exhibited marked 
A

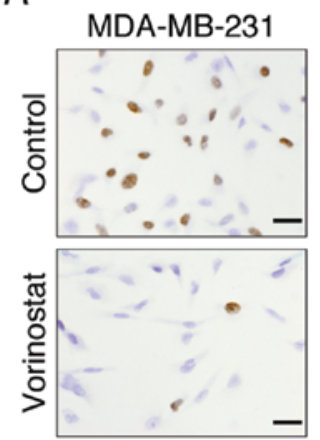

B

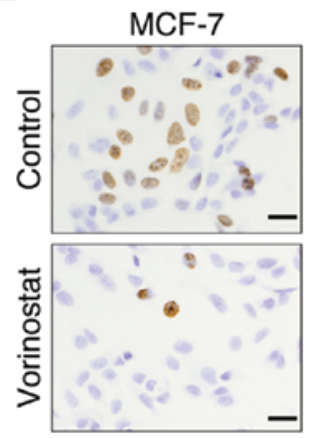

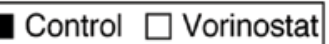
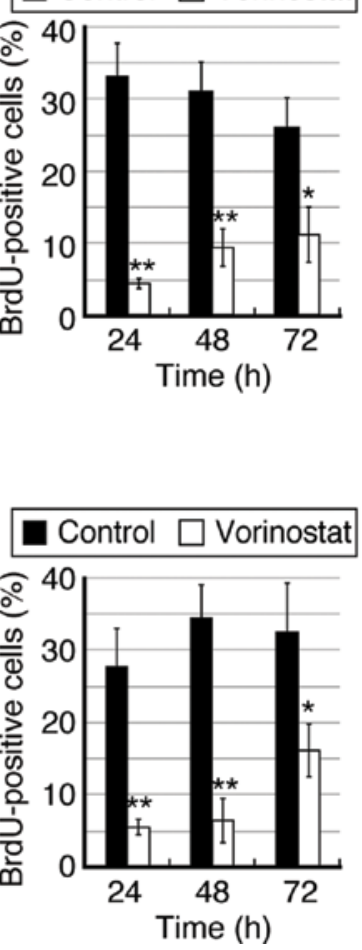

Figure 1. Effect of vorinostat on BrdU incorporation in MDA-MB-231 and MCF-7 human breast cancer cells. MDA-MB-231 (A) and MCF-7 (B) cells were treated with the $\mathrm{IC}_{50}$ of vorinostat (5.0 and $2.5 \mu \mathrm{M}$, respectively) for $72 \mathrm{~h}$. Representative micrographs for BrdU staining at $24 \mathrm{~h}$ after treatment with vorinostat are shown. BrdU-labeling indices were determined at the indicated time points as described in Materials and methods. Columns indicate the percent of BrdU-positive cells in five fields of 1,000 cells total from each separate experiment. ${ }^{*} \mathrm{P}<0.05$ and ${ }^{* *} \mathrm{P}<0.01$ as compared with untreated control cells at each time point.

upregulation of p21 mRNA, which was correlated with its protein level (Fig. 2A and C, left panel). MCF-7 cells showed a modest increase in p21 mRNA at 48-72 h after vorinostat treatment, whereas only a slight increase was observed at $24 \mathrm{~h}$ (Fig. 2B). Interestingly, a marked increase in p21 protein was observed in MCF-7 cells $24 \mathrm{~h}$ after vorinostat treatment, and the increased protein level persisted until $72 \mathrm{~h}$ (Fig. 2C, right panel). Moreover, MDA-MB-231 and MCF-7 cells displayed a remarkable increase in the $\mathrm{p} 27$ protein level after vorinostat treatment (24-72 h), despite no significant increase in the p27 mRNA level (Fig. 2). These results indicate that vorinostat may control p27 and p21 protein levels through post-translational regulation in these cell lines.

Vorinostat downregulates Skp2 and Cks1 expression in human breast cancer cells. We next focused our attention on the expression of Skp2 and Cks1, which are responsible for p21 and p27 stability (19). Vorinostat significantly reduced the Skp2 and Cks1 mRNA levels and the concomitant protein levels in MDA-MB-231 and MCF-7 cells (Fig. 3). Skp1, another component of the $\mathrm{SCF}^{\text {Skp2-Cks1 }}$ ubiquitin ligase complex, did not show any change in response to vorinostat (Fig. 3C). The vorinostat-induced decreases in the Skp2 and Cks1 levels paralleled the reduction of BrdU-incorporation, which was

A

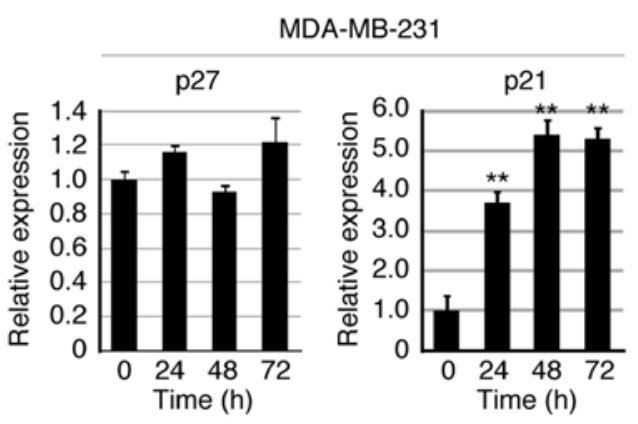

B

MCF-7
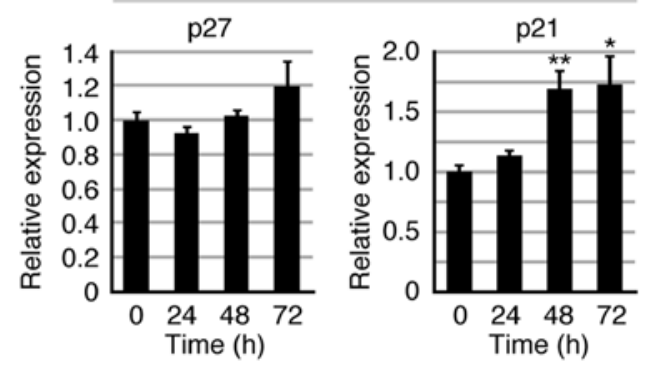

C
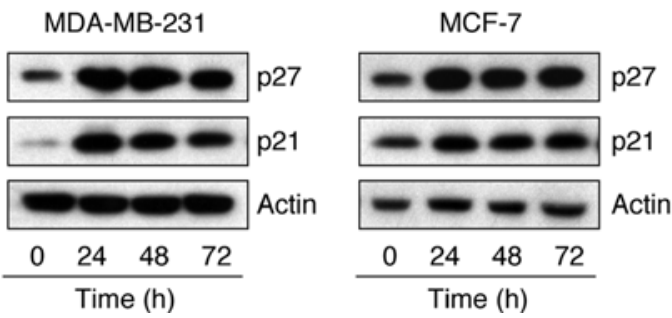

Figure 2. Effect of vorinostat on p27 and p21 expression in human breast cancer cells. (A and B) mRNA expression of p27 and p21 was analyzed by real-time PCR and was normalized to the level of $\beta$-actin, and the expression level is shown relative to the control as 1 . Data represent the mean $\pm \mathrm{SD}$ of three independent experiments. ${ }^{*} \mathrm{P}<0.05$ and ${ }^{* *} \mathrm{P}<0.01$ as compared with untreated control cells. (C) Lysates were prepared from MDA-MB-231 and MCF-7 cells at the indicated time points after vorinostat treatment. Western blot analysis was performed by using antibodies specific to the indicated proteins.

indicative of the inhibition of S-phase entry (Figs. 1 and 3). These results suggest that the post-translational accumulation of p27 and p21 through Skp2 and Cks1 downregulation may contribute to vorinostat-induced growth arrest.

Vorinostat enhances the stability of p27 and p21 proteins. To determine whether the accumulation of p27 and p21 in response to vorinostat is due to enhanced protein stability, we further evaluated the half-life of p27 and p21 proteins by using $\mathrm{CHX}$, an inhibitor of protein synthesis. As shown in Fig. 4, the protein stability of p27 and p21 in MDA-MB-231 and MCF-7 cells was significantly enhanced by vorinostat treatment as compared to untreated control cells. Interestingly, MDA-MB-231 cells exhibited a higher resistance to p27 protein degradation as compared to $\mathrm{p} 21$ degradation; $70 \%$ in vorinostat-treated cells vs. $\sim 25 \%$ in untreated control cells for p27 and $\sim 35 \%$ (vorinostat) vs. $~ 15 \%$ (control) for p21 (Fig. 4A and B). In contrast, MCF-7 cells exhibited more resistance to p21 protein degradation as compared to p27 protein degradation; $\sim 65 \%$ (vorinostat) vs. $\sim 35 \%$ (control) for $\mathrm{p} 27$ and $\sim 80 \%$ (vorinostat) vs. $\sim 30 \%$ (control) for p21 (Fig. 4C and D). 
A

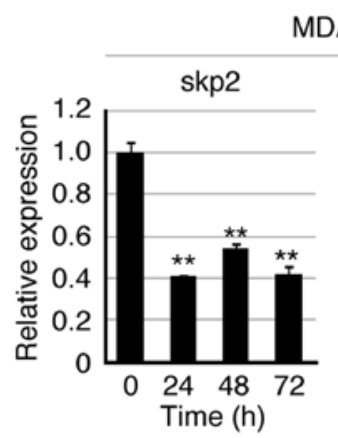

MDA-MB-231

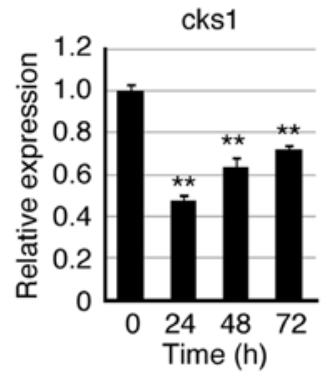

B

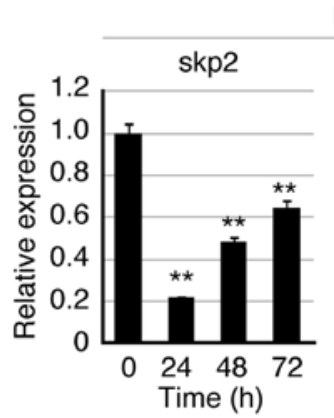

MCF-7

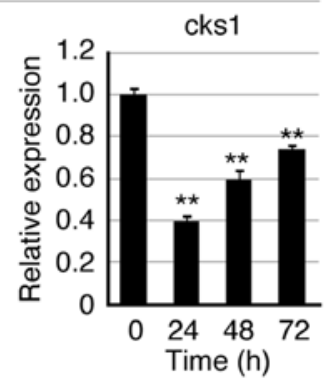

C
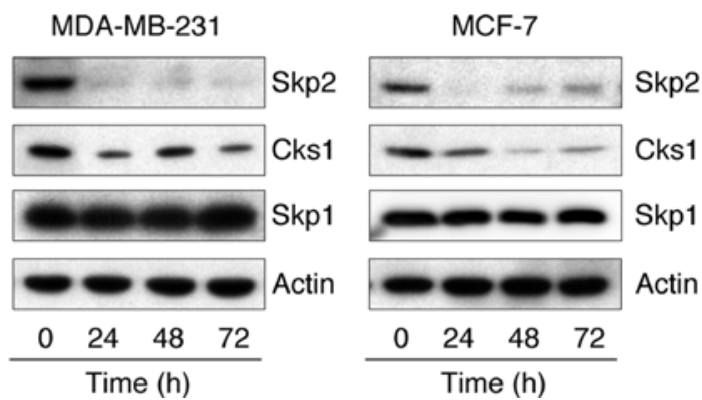

Figure 3. Effect of vorinostat on Skp2 and Cks1 expression in human breast cancer cells. (A and B) mRNA expression of Skp2 and Cks1 was analyzed by real-time PCR analysis and normalized to the level of $\beta$-actin. The expression level is shown relative to the control as 1 (A and B). Data represent the mean $\pm \mathrm{SD}$ of three independent experiments. ${ }^{*} \mathrm{P}<0.05$ and ${ }^{* * *} \mathrm{P}<0.01$ as compared with untreated control cells. (C) Lysates were prepared from MDA-MB-231 and MCF-7 cells at the indicated time points after vorinostat treatment. Western blot analysis was performed with antibodies specific to the indicated proteins.

Enhanced expression of Skp2 and Cks1 induces G1-S phase transition in vorinostat-treated MDA-MB-231 cells. Due to the fact that we observed that vorinostat-induced growth inhibition was correlated with the enhanced stability of p27 and p21 proteins and decreases in the Skp2 and Cks1 levels, we hypothesized that the maintenance of Skp2 and Cks1 expression may attenuate the anti-proliferative effect of vorinostat. To confirm this hypothesis, we examined the impact of enforced expression of Skp2 and Cks1 upon p27 and p21 protein levels and cell-cycle distribution after vorinostat treatment in MDA-MB-231 cells. As shown in Fig. 5A, induction of p27 and p21 by vorinostat was diminished by the enforced expression of Skp2 and Cks1. Cell-cycle analysis revealed that vorinostat-induced $\mathrm{S}$-phase reduction was markedly recovered by Skp2 and Cks1 expression (Fig. 5B). These results indicate that the decrease in Skp2 and Cks1 by vorinostat may be involved in growth inhibition through the regulation of p27 and $\mathrm{p} 21$ protein abundance.

\section{Discussion}

CKIs, such as p27 and p21, play a critical role in HDACiinduced growth arrest (11). Despite the importance of p27 and p21 protein abundance control for cell-cycle progression, information on post-translational regulation of these proteins by HDACi is limited $(20,21)$.

Earlier studies demonstrated that HDAC inhibition by trichostatin A (TSA) induces post-translational accumulation of p27 in human pancreatic cancer cells (21) and rodent fibroblasts (20). In agreement with these studies, we observed an elevated p27 protein level without any changes in its mRNA levels after vorinostat treatment in MDA-MB-231 and MCF-7 human breast cancer cells (Fig. 2). Our results also showed that MCF-7 cells exhibited a marked increase in the p21 protein level as compared to the p21 mRNA levels after vorinostat treatment (Fig. $2 \mathrm{~B}$ and $\mathrm{C}$ ). These results indicate that vorinostat controlled p27 and p21 protein abundance at the post-translational level. p27 and p21 are substrates for $\mathrm{SCF}^{\text {Skp2-Cks1 }}$ ubiquitin ligase through phosphorylation by the cyclin-CDK complex. SCF ${ }^{\text {Skp2-Cks1 }}$ consequently catalyzes the polyubiquitination of p27 and p21 and tags both proteins for proteasomal degradation (22-24). As expected, vorinostat downregulated Skp2 and Cks1 mRNA expression and their concomitant protein levels in MDA-MB-231 and MCF-7 cells (Fig. 3). It has been reported that transcription of $S k p 2$ is mediated through HDAC3 recruitment to the $S k p 2$ promoter region (21). Vorinostat can inhibit multiple HDACs including HDAC3. Downregulation of Skp2 might be due to the inhibitory effect of vorinostat on HDAC3. Alao et al reported that treatment of MCF-7 cells with TSA transiently increased Skp2 expression (from 4 to $6 \mathrm{~h}$ after treatment) (25). However, we could not detect an increase in the Skp2 mRNA or protein level by vorinostat at 1,3 and $6 \mathrm{~h}$ in MCF-7 cells (data not shown). Although both TSA and vorinostat induce growth arrest in MCF-7 cells, the difference in Skp2 expression may be due to the different concentration and type of chemicals. The precise mechanisms seem to be complex and controversial.

To the best of our knowledge, this is the first report of Cks1 downregulation by vorinostat in breast cancer cells. Cks1 is required for efficient ubiquitination and recognition of p27 and p21 (26,27); therefore, downregulation of Cks1 by vorinostat would contribute to p27 and p21 protein stability. In fact, vorinostat-mediated decreases in Skp2 and Cks1 levels coincided with increased resistance of p27 and p21 to protein degradation in MDA-MB-231 and MCF-7 cells (Figs. 2 and 4). Interestingly, there is a selective stability of p27 and p21 proteins between MDA-MB-231 and MCF-7 cells (Fig. 4). Vorinostat induced cell-cycle arrest at the G2/M phase in MDA-MB-231 cells (Fig. 5) and at the G1 phase in MCF-7 cells (28). Vorinostat induces cell-cycle arrest at the G1 or the G2/M boundary in a cell-type dependent and/or dose-related fashion (11). We consider it likely that the selective stability of p27 or p21 by vorinostat in MDA-MB-231 and MCF-7 cells may partially explain the different points of cell-cycle arrest by HDACi.

Signoretti et al demonstrated that the forced expression of Skp2 in MCF-7 cells conferred resistance to tamoxifeninduced S-phase reduction (6). Since forced expression of 
A

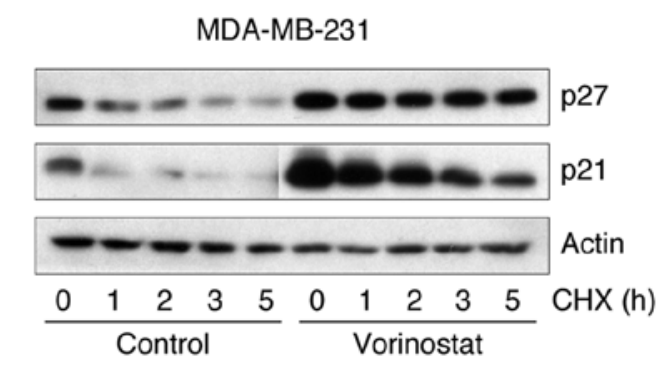

C

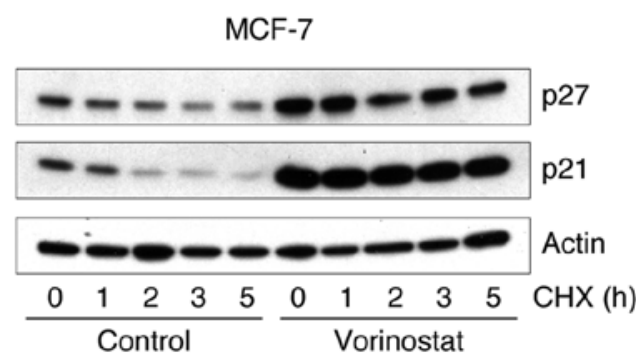

B

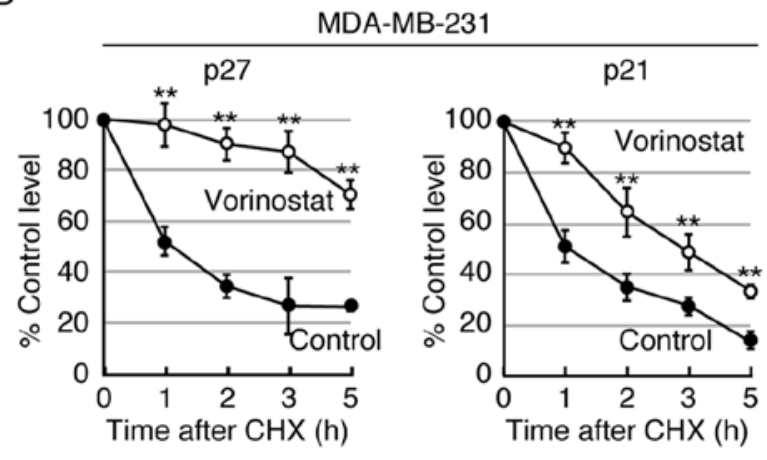

D

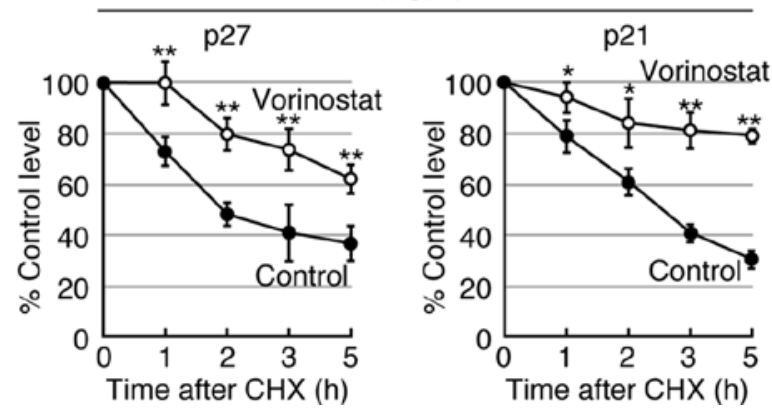

Figure 4. Vorinostat enhances p27 and p21 protein stability in breast cancer cells. (A and B) MDA-MB-231 and (C and D) MCF-7 cells were treated with the $\mathrm{IC}_{50}$ of vorinostat (5.0 and $2.5 \mu \mathrm{M}$, respectively) for $24 \mathrm{~h}$, and then $10 \mu \mathrm{g} / \mathrm{ml} \mathrm{CHX}$ was added. (A and C) At the indicated time points after CHX treatment, cell lysates were prepared and western blot analysis was performed with antibodies specific to the indicated proteins. (B and D) Band intensity was normalized to actin, and the protein level is shown relative to the $\mathrm{t}=0$ controls. Data represent the mean \pm SD of three independent experiments. ${ }^{*} \mathrm{P}<0.05$ and ${ }^{* * *} \mathrm{P}<0.01$ as compared with control cells at each time point.

A

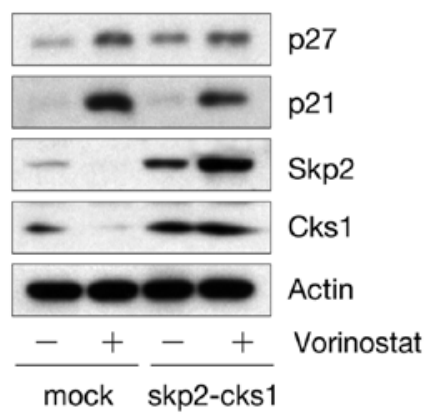

B

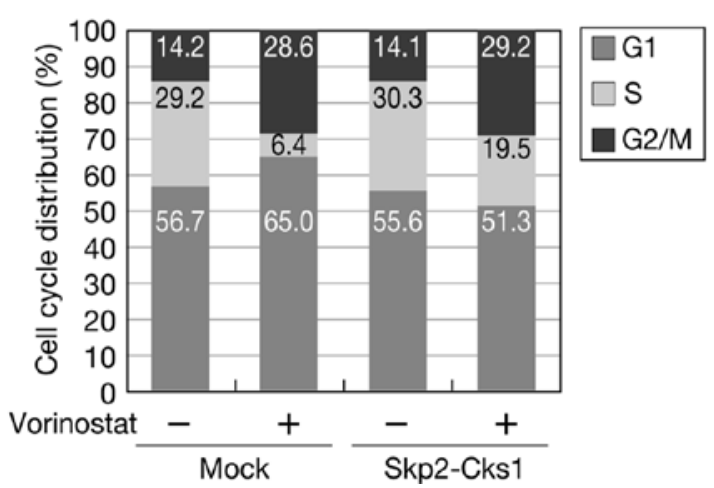

Figure 5. Effect of Skp2 and Cks1 on the cell-cycle distribution after vorinostat-induced growth arrest of MDA-MB-231 cells. At $24 \mathrm{~h}$ after transfection of Skp2 and Cks1, MDA-MB-231 cells were treated with $5.0 \mu \mathrm{M}$ vorinostat for $24 \mathrm{~h}$. (A) Cell lysates were prepared and western blot analysis was performed with antibodies specific to the indicated proteins. (B) Cell-cycle distribution was analyzed by FACS as described in Materials and methods.
Skp2 alone in MDA-MB-231 cells showed little, if any, effect on p27 or p21 protein levels in response to vorinostat treatment (data not shown), we introduced both Skp2 and Cks1 in MDA-MB-231 cells to examine the impact of these molecules on vorinostat-induced growth inhibition (Fig. 5). Similar to the results demonstrated by Signoretti et al (6), the vorinostat-induced $S$-phase reduction was markedly recovered by Skp2 and Cks1 expression in MDA-MB-231 cells (Fig. 5B). Interestingly, the forced expression of Skp2 and Cks1 did not affect cell proliferation after vorinostat treatment as compared with vector control cells. This might be due to the decrease in cyclin A, cyclin B1, cyclin E, Cdk1, Cdk2 and Cdk6 by vorinostat treatment (data not shown).

Collectively, our data imply that not only the transcriptional activation of $\mathrm{p} 21$, but also the enhanced protein stability of p27 and p21 through Skp2 and Cks1 regulation, may play a pivotal role in the vorinostat-induced growth arrest of human breast cancer cells. Moreover, Skp2 and Cks1 are the targets of vorinostat in breast cancer cells regardless of ER status. Further investigations are needed to clarify how HDAC inhibition suppresses Skp2 and Cks1 expression. Such findings could provide novel insights into effective therapies for breast cancer and other malignancies that overexpress Skp2 and Cks1.

\section{Acknowledgements}

We thank Dr Michele Pagano (NYU Cancer Institute, New York University, NY) for generously providing the expression 
plasmid pcDNA-FLAG-Skp2 and pcDNA-FLAG-Cks1. We thank Ms. T. Akamatsu for technical assistance. This study was supported by a Grant-in-Aid for Young Scientists (B) (20790940 and 22791263) (N.U.).

\section{References}

1. Sherr CJ and Roberts JM: CDK inhibitors: positive and negative regulators of G1-phase progression. Genes Dev 13: 1501-1512, 1999.

2. Reed SI: Ratchets and clocks: the cell cycle, ubiquitylation and protein turnover. Nat Rev Mol Cell Biol 4: 855-864, 2003.

3. Catzavelos C, Bhattacharya N, Ung YC, et al: Decreased levels of the cell-cycle inhibitor p27Kip1 protein: prognostic implications in primary breast cancer. Nat Med 3: 227-230, 1997.

4. Slingerland J and Pagano M: Regulation of the cdk inhibitor p27 and its deregulation in cancer. J Cell Physiol 183: 10-17, 2000.

5. Hershko DD: Oncogenic properties and prognostic implications of the ubiquitin ligase Skp2 in cancer. Cancer 112: 1415-1424, 2008.

6. Signoretti S, Di Marcotullio L, Richardson A, et al: Oncogenic role of the ubiquitin ligase subunit Skp2 in human breast cancer J Clin Invest 110: 633-641, 2002.

7. Slotky M, Shapira M, Ben-Izhak O, et al: The expression of the ubiquitin ligase subunit Cks1 in human breast cancer. Breast Cancer Res 7: R737-R744, 2005.

8. Sonoda $\mathrm{H}$, Inoue $\mathrm{H}$, Ogawa $\mathrm{K}$, Utsunomiya T, Masuda TA and Mori M: Significance of skp2 expression in primary breast cancer. Clin Cancer Res 12: 1215-1220, 2006.

9. Traub F, Mengel M, Luck HJ, Kreipe HH and von Wasielewski R: Prognostic impact of Skp2 and p27 in human breast cancer. Breast Cancer Res Treat 99: 185-191, 2006.

10. Bolden JE, Peart MJ and Johnstone RW: Anticancer activities of histone deacetylase inhibitors. Nat Rev Drug Discov 5: 769-784, 2006.

11. Richon VM, Garcia-Vargas J and Hardwick JS: Development of vorinostat: current applications and future perspectives for cancer therapy. Cancer Lett 280: 201-210, 2009.

12. Huang L, Sowa Y, Sakai T and Pardee AB: Activation of the p21WAF1/CIP1 promoter independent of p53 by the histone deacetylase inhibitor suberoylanilide hydroxamic acid (SAHA) through the Sp1 sites. Oncogene 19: 5712-5719, 2000.

13. Bali P, Pranpat M, Swaby R, et al: Activity of suberoylanilide hydroxamic Acid against human breast cancer cells with amplification of her-2. Clin Cancer Res 11: 6382-6389, 2005

14. Huang L and Pardee AB: Suberoylanilide hydroxamic acid as a potential therapeutic agent for human breast cancer treatment. Mol Med 6: 849-866, 2000.

15. Uehara N, Kanematsu S, Miki H, Yoshizawa K and Tsubura A: Requirement of p38 MAPK for a cell-death pathway triggered by vorinostat in MDA-MB-231 human breast cancer cells. Cancer Lett 315: 112-121, 2012.
16. Uehara N, Unami A, Kiyozuka Y, Shikata N, Oishi Y and Tsubura A: Parous mammary glands exhibit distinct alterations in gene expression and proliferation responsiveness to carcinogenic stimuli in Lewis rats. Oncol Rep 15: 903-911, 2006.

17. Uehara N, Matsuoka Y and Tsubura A: Mesothelin promotes anchorage-independent growth and prevents anoikis via extracellular signal-regulated kinase signaling pathway in human breast cancer cells. Mol Cancer Res 6: 186-193, 2008

18. Kanematsu S, Uehara N, Miki H, et al: Autophagy inhibition enhances sulforaphane-induced apoptosis in human breast cancer cells. Anticancer Res 30: 3381-3390, 2010.

19. Nakayama KI and Nakayama K: Regulation of the cell cycle by SCF-type ubiquitin ligases. Semin Cell Dev Biol 16: 323-333, 2005.

20. Chen JS and Faller DV: Histone deacetylase inhibition-mediated post-translational elevation of p27KIP1 protein levels is required for G1 arrest in fibroblasts. J Cell Physiol 202: 87-99, 2005.

21. Schneider G, Reichert M, Saur D, Hamacher R, Fritsch R and Schmid RM: HDAC3 is linked to cell cycle machinery in $\mathrm{MiaPaCa} 2$ cells by regulating transcription of skp2. Cell Prolif 40: 522-531, 2007.

22. Bornstein G, Bloom J, Sitry-Shevah D, Nakayama K, Pagano M and Hershko A: Role of the SCFSkp2 ubiquitin ligase in the degradation of p21Cip1 in S phase. J Biol Chem 278: 25752-25757, 2003.

23. Carrano AC, Eytan E, Hershko A and Pagano M: SKP2 is required for ubiquitin-mediated degradation of the $\mathrm{CDK}$ inhibitor p27. Nat Cell Biol 1: 193-199, 1999.

24. Tsvetkov LM, Yeh KH, Lee SJ, Sun H and Zhang H: p27(Kip1) ubiquitination and degradation is regulated by the $\mathrm{SCF}(\mathrm{Skp} 2)$ complex through phosphorylated Thr187 in p27. Curr Biol 9: 661-664, 1999.

25. Alao JP, Lam EW, Ali S, et al: Histone deacetylase inhibitor trichostatin A represses estrogen receptor alpha-dependent transcription and promotes proteasomal degradation of cyclin D1 in human breast carcinoma cell lines. Clin Cancer Res 10: 8094-8104, 2004.

26. Ganoth D, Bornstein G, Ko TK, Larsen B, Tyers M, Pagano M and Hershko A: The cell-cycle regulatory protein Cks1 is required for $\mathrm{SCF}(\mathrm{Skp} 2)$-mediated ubiquitinylation of $\mathrm{p} 27$. Nat Cell Biol 3: 321-324, 2001.

27. Spruck C, Strohmaier H, Watson M, et al: A CDK-independent function of mammalian Cks1: targeting of $\mathrm{SCF}(\mathrm{Skp} 2)$ to the CDK inhibitor p27Kip1. Mol Cell 7: 639-650, 2001.

28. Munster PN, Troso-Sandoval T, Rosen N, Rifkind R, Marks PA and Richon VM: The histone deacetylase inhibitor suberoylanilide hydroxamic acid induces differentiation of human breast cancer cells. Cancer Res 61: 8492-8497, 2001. 